\title{
Mobile Controlled Automated wheelchair for Disabilities
}

\author{
Srinivasan Selvaraj, A.Ganasekar, Pacha Shobha Rani, Dr.P.Ezhumalai
}

\begin{abstract}
As the usage of the Android smart phones has been considerably increasing, a lot of applications have been developed for the benefits of mobile users. In the past, many applications have been designed aiming to help physically disabled persons. This paper presents an android application which providers several options for controlling the movement of wheelchairs effectively. The proposed application enables People with Disabilities (PWDs) to operate the wheel chair with minimum effort. Apart from voice commands, the proposed application detects and measures the tilt change, and moves the wheelchair based on the degree of the tilt. It also provides a soft joystick as in mobile games to ease the operation of the wheelchairs. Furthermore, sensors that are fixed in the wheelchair can detect and avoid obstacles when the chair is on the move. Hence, it ensures the safety while using the wheelchairs. The proposed application will help both physically challenged persons and elders to operate the wheelchairs more comfortably.
\end{abstract}

Keywords: Android, Wheel chair, physically disabled persons, voice commands.

\section{INTRODUCTION}

The number of disabled people has been increasing significantly as the world population grows. It is estimated that about $15 \%$ of world population have some kind of disability. Though some people are born with disability, many people become disabled due to injuries and health problems. Perhaps, people with disabilities (PWD) depends non-disabled people including family members for their mobility. Wheelchairs are one of the key assistive devices for PWD across the world. Generally, wheelchairs are grouped into five categories, namely: Manual wheelchairs, Attendant-propelled chairs, rigid frame wheelchair, folding frame wheelchair and motorized wheelchair/ power chair/ electric-powered wheelchair. Among them, electric-powered wheelchair is more suitable for PWD as it reduces their dependence on their assistants. Moreover, it is suited for people with physical and cognitive skills [1]. As it facilitates high mobility, it enables PWD to carry out their day to day activities with ease. Additionally, wheelchairs are also utilized by people with cardiovascular and fatigue-based

Revised Manuscript Received on November 27, 2019

* Correspondence Author

*Srinivasan Selvaraj*,Department of CSE, RMD Engineering College, Chennai, INDIA. Email: ssn.cse@ rmd.ac.in

A.Ganasekar, Department of CSE, RMD Engineering College, Chennai, INDIA. Email: : ags.cse@rmd.ac.in

Pacha Shoba Rani, Department of CSE, RMD Engineering College, Chennai, INDIA. Email: : ags.cse@rmd.ac.in

Dr P. Ezhumalai, Prof \& Head, Department of CSE, RMD Engineering College, Chennai, INDIA. Email: : ags.cse@rmd.ac.in illness [2][3].However, 40 percent of PWDs like people with low vision found difficulties in operating electric wheelchairs. To resolve those difficulties, smart wheelchairs have been developed and used by PWD in the last few decades.

Basically, a smart wheelchair consists of a power chair with a set of sensors and computerized program. Sensors are placed on a mobile robot which is subsequently fixed with the wheelchair. Smart power wheelchairs have the capability of performing navigation automatically. It determines the navigation paths based on the environment and accomplish the navigation task without the user intervention. Perhaps, the motion of wheelchairs is controlled by the computerized program. One common observation about smart wheelchair is that the mobile robot in the smart wheelchairs has to finalize the path based on the internal map of the surroundings. Therefore such wheelchairs become useless in the unknown environments and they fail to detect unplanned obstacles [4]. In another type of smart wheelchairs, the user decides the path to reach the destination. and the user control the movement of the chair through different inputs. Generally, inputs devices such as joy stick, voice, or electrooculography activities such as eye movement, finger movement are used to control navigation [5][6][7]. Zhang et al [14] developed Curtin University Brailler (CUB), a Personal Digital Assistant (PDA), to help visually impaired people. However, as the smart phones have gained popularity, people have started to use them to carry out dayto- day activates. Since smart phones particularly android smart phones are affordable, they have been widely used

This paper presents a new android application to operate smart wheelchairs. The proposed application provides multiple interfaces by which the user controls wheel chairs by voice, tilt and mobile gamepad. The unique aspect of the proposed work is that it uses the built-in sensors and software to accelerate or decelerate the wheels of the wheelchairs. In addition, an array of sensors embedded in the mobile robot is used to explore the surroundings. It can also detect obstacles. Hence, it assures the ultimate travel experience to wheel-chair reliant people.

This paper is organized as follows. Section 2 presents the related work. Section 3 presents the insight of the proposed system and Section 4 explains how the proposed system is implemented. Finally, Section 5 concludes.

\section{LITERATURE SURVEY}

Simpson did a detailed survey on smart wheelchairs in $[4,17]$. He also analysed the impact of smart wheelchairs on the life of People with Disabilities in [1]. It is found that various efforts have been taken to address the problems of people

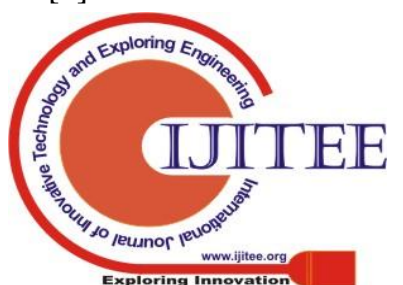


with different disabilities in the literature. But, this section focuses on works that have used smart devices to control wheelchairs only.

Shaheen and Umamakeswari developed an embedded system for dump, blind and paralysis affected people to operate the wheelchairs. They have used gloves made of flex sensors to sense the finger movements of paralysed person to move the wheelchair in the desired direction [6]. Sharadda and Narendra developed an android application which controls the wheelchair movement through voice and gesture moves. They aimed to replace the traditional joystick which is difficult to operate by people with severe disabilities. Their system could avoid obstacles with the help of IR sensors[10]. Gautham et al designed another android application for people who suffered with paralysed attack to control the wheelchair using eye blink sensors [13]. Similarly, Pramila et al developed an application which can operate the wheelchair through voice and keypad. However, most of the existing android applications are not incorporated any mechanism to control the speed of wheelchair movements and provide a standard 4X4 keypad interface. The android application proposed in [16] allowed PWD to move to the desired location like Hall, kitchen without the help of caregivers. Moreover this techiques direct the wheelchair using line following algorithm where the PWDs have no control over the movement. Shen et al have designed smart wheelchair which is operated by tele-presence robot. Their system also provides limited mobility to the user through a joystick interface [15]. Unlike the existing applications, the proposed application provides multiple interfaces to operate smart wheelchairs. Additionally, it allows PWDs to customize the commands in order to improve their comfort.

\section{THE PROPOSED SYSTEM}

The proposed system consists of two parts, namely: sensory part and android application. This section explains the role of various hardware devices used in the proposed system. As shown in the Fig. 1 the android application and the sensory part which is attached to the wheelchair are connected through Bluetooth. When a disabled person wants to move, he/she uses the application to issue a command. The command is converted into a textual message using inbuilt speech recognition software. The message is passed to the Bluetooth module of the Android phone which transfers the same to the sensory unit in the wheelchair. The program runs on the microcontroller receives the message and direct the wheelchair towards the desired location. The Light Sensor (LDR) on the sensory unit helps to detect obstacles in order to avoid them. Similarly, the disabled user can operate the wheelchair using the MEMS technology. When the screen is tilted horizontally, the accelerometer detects the orientation change and measure the speed of the acceleration. It moves the wheel chair according to the acceleration.

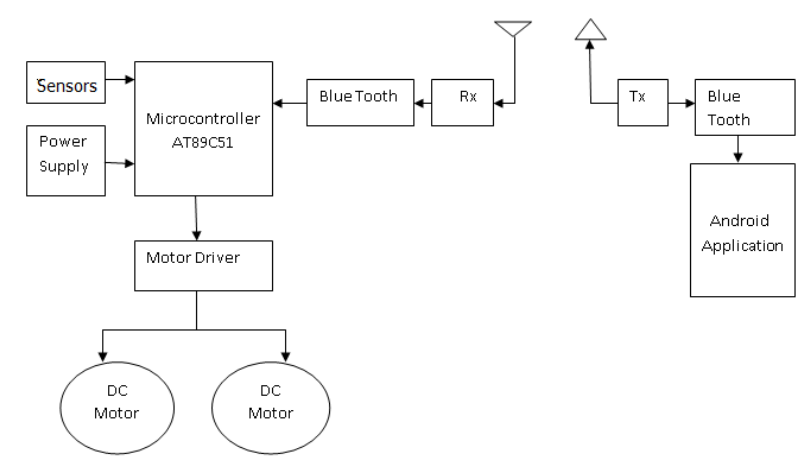

Fig.1 SYSTEM ARCHITECTURE

The Android application provides a user friendly, customizable interface by which PWDs can move the wheelchair in any directions (forward, backward, left and right). It allows the user to use voice, gesture and keypad to move the chair. When a person with disabilities issues a voice command, internal mic of the phone receives the command and converts the voice command into text using inbuilt speech recognition in the android phone. For instance, if the user wants to move in left direction, the user has to tell the command "LEFT". Perhaps, voice commands can be recognized by the built in speech reorganization module only when the phone is closer to the user. So the application provides few more options. It provides interface with soft keypad which simplifies locomotion of the users. Similarly, the user can control the movement of the wheelchair through tilting. It uses the MEMS (Micro-Electrical Mechanical Systems) Accelerometer in the smart phone to control the speed of movement of the wheelchair. Moreover, the application allows customizing controls so that the users can set commands and selects buttons based on their preference.

\section{SYSTEM DESIGN AND IMPLEMENTATION}

The proposed system uses a PIC 16F Microcontroller (Ardunio), motor driver( LC293D), a Bluetooth module for communication(HC05), a light sensor (LDR), DC Wheel motor of 5rpm, Precision Shaft Potentiometer, Power Supply- 12V and MEMS Accelerometer Precision Shaft Potentiometer and Android Phone (Smart Phone). The Keil IDE is used to $C$ programs for the microcontroller while Eagle is used for PCB Design.

The following Fig. 2 and Fig. 3 show the hardware setup of the smart wheel chair for the people with disabilities.

The screenshots of the android application is shown form Fig.4 to Fig.9. Fig.4 shows the main menu of the application showing all alternative options to operate the wheelchair. There are four options, namely: Vehicle Mode(Tilt Control), Fader Mode (Voice Control), Controller Mode (Keypad and Joystick), and Terminal Mode available to the user. When the user selects the Fader Mode, a new interface as shown in Fig. 5 for receiving the voice commands is presented to the user. The user can issue voice commands like RIGHT, LEFT to direct the chair in a particular direction. 


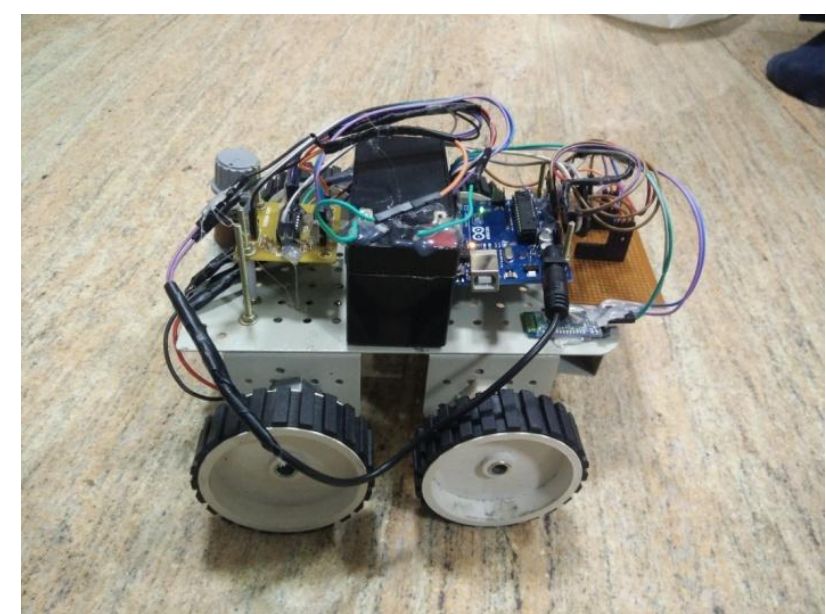

Fig.2 Side View

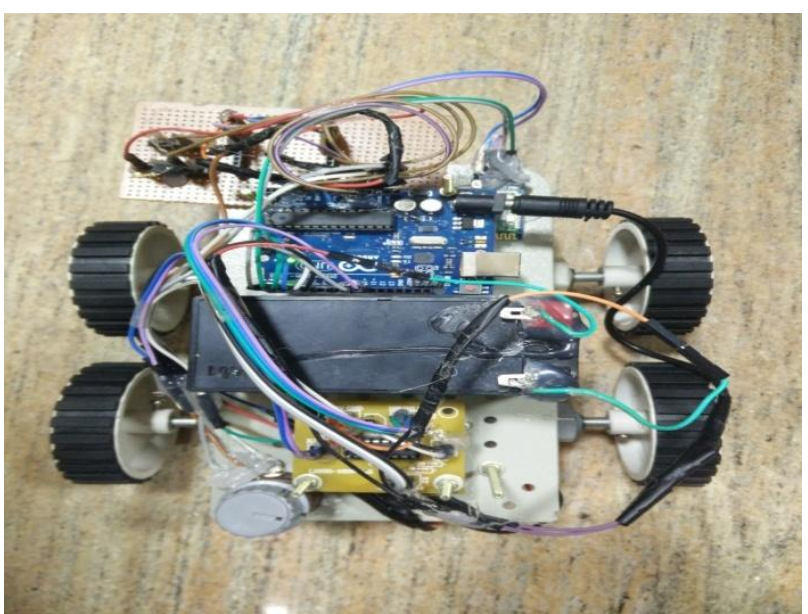

Fig 3. Top View

Fig.6 shows two interfaces available in the Controller mode. When the user selects this mode, the user can control the movements of the wheelchair through array keys and a joystick as in mobile games. Fig.7 depicts the terminal mode interface by which the user can customize the functionalities associated with the keys based on his convenience.

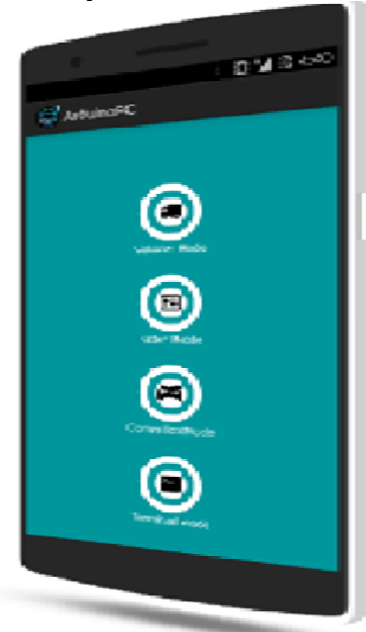

Fig 3. Menu

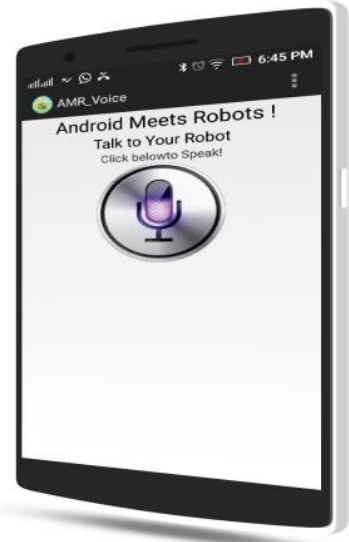

Fig. 4 Voice Module Interface

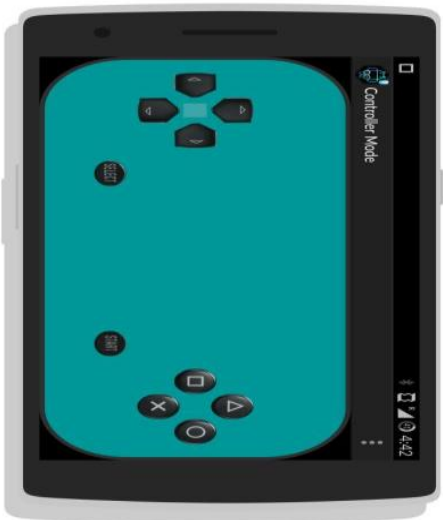

Fig. 5 Keypad and Joystick Interface

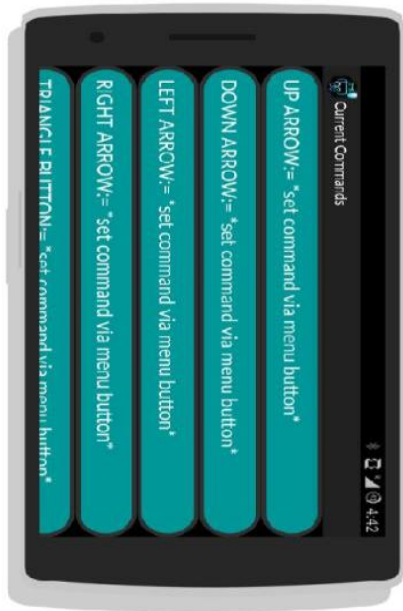

Fig.6 Command Mode Interface

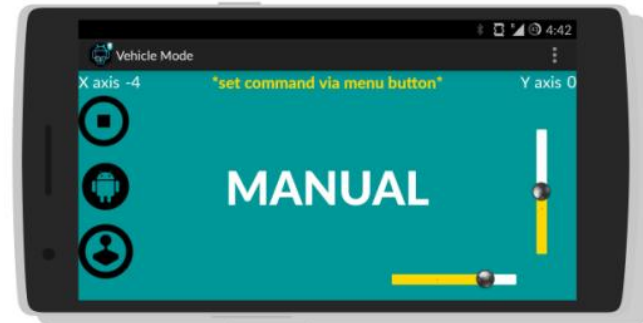

Fig.7 Tilt Control Interface - To move on RIGHT direction 


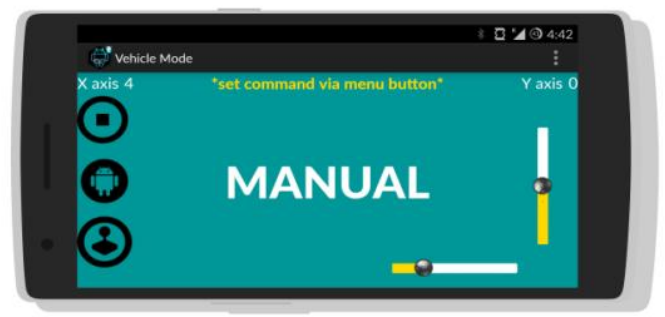

Fig.9. Tilt Control Interface - To move on LEFT direction

The Fig. 8 and Fig. 9 shows the Vehicle Mode interface. It shows how the user can move the wheel chair on the right direction and left direction and control the speed of the wheelchair.

\section{CONCLUSION}

As the number of people with disabilities has been constantly increased, there is need to develop different assistive tools for those people. Perhaps, many software applications have been developed and used by the people who are deaf, hearing impaired, blind or language disorders and paralyzed. In this paper, a new android application is designed to assist the physically challenged people. The proposed application controls the wheel chair through voice commands and gesture moves. As the application does not need any additional accessories, it is easy to operate. Perhaps, it provides multiple interfaces that are customizable and better speed control. It makes the life of both PWD and elderly people easier.

\section{REFERENCES}

1. Simpson RC, LoPresti EF, Cooper RA,"How many people would benefit from a smart wheelchair?", Journal of Rehabilitation Research \& Development, Vol.45, No:1, pp: 53-72, 2008

2. Bedaf S, Gelderblom G J, De Witte L.," Overview and Categorization of Robots Supporting Independent Living of Elderly People: What Activities Do They Support and How Far Have They Developed[J]", Assistive Technology, Vol: 27, No:2,pp: 88-100,2015

3. Tom Carlson , Yiannis Demiri,'Increasing Robotic Wheelchair Safety With Collaborative Control: Evidence from Secondary Task Experiments" Proc. Of Intl. Conf. on Robotics and Automation,pp: 5582-558, 2010

4. Simpson RC," Smart wheelchairs: A literature review, Journal of Rehabilitation Research \& Development, Vol:42, No:4, :pp:423-436, 2005. 5. Cheng, W-C., \& Chiang, C-C, "The development of the automatic lane following navigation system for the intelligent wheelchair" In Proceedings of IEEE international conference on fuzzy systems (FUZZ-IEEE 2011) pp. 1946-1952, 2011.

6. S. Shaheen, A. Umamakeswari," ARM Based 3-in-1 Device for People with Disabilities", Journal of Artificial Intelligence, Vol: 6(1), pp:75-81, 2013

7. K. Rajeswari, E. Jeevitha and V. K. G. K. Selvi, "“Virtual Voice" — The voice for the dumb,", Proc. Of IEEE International Conference on Computational Intelligence and Computing Research (ICCIC), Coimbatore, pp. $1-3,2010$

8. C. Krishnaswamy and G. Srikkanth, "Dynamic Acoustic for Dumb Using Embedded Interface (DADEI)," Proc of Second International Conference on Computer Modeling and Simulation, pp. 525-527, 2010,.

9. Lu, Tao, et al. "An embedded control system for intelligent wheelchair", Proc. Of 27th IEEE Annual International Conference of the Engineering in Medicine and Biology Society IEEE-EMBS, Vol.5,pp:5036-5039, 2005

10. Khadilkar, Shraddha Uddhav, and Narendra Wagdarikar. "Android phone controlled voice, gesture and touch screen operated smart wheelchair", Proc. Of IEEE International Conference on Pervasive Computing (ICPC), 2015
11. Fezari, Mohamed, Mounir Bousbia-salah, and Mouldi Bedda. "Voice and Sensor for More Security on an Electric Wheelchair." Proc. Of $2^{\text {nd }}$ Int'l Conference on Information and Communication Technologies ICTTA'06, Vol. 1,pp:854-858, 2006.

12. Pramila Kupkar, Prajakta Pandit, Nikita Dhamdhere \& P.P. Jadhav,"Android Controlled Wheel Chair", Imperial Journal of Interdisciplinary Research (IJIR), Vol-2, Issue-6, pp: 1219-1222, 2016,

13. Gautham G, Kiran Kumar C , Manjunath S D \& Mohammed Moin Pasha Khaleel," Wheel Chair Movement Control Using Eye Blink Sensors and Smart Phone", Imperial Journal of Interdisciplinary Research (IJIR), Vol-2, Issue-7, pp: 842-846, 2016

14. X. Zhang, C. Ortega-Sanchez and I. Murray, "Reconfigurable PDA for the Visually Impaired Using FPGAs," Proc. Of International Conference on Reconfigurable Computing and FPGAs, pp. 1-6, 2008

15. Jiajun Shen, Bin Xu, Mingtao Pei, Yunde Jia," A Tele-Presence Wheelchair for Elderly People", http://arxiv.org/abs/1601.06005v1

16. S.Shaheen, A.Umamakeswari," Intelligent Wheelchair for People with Disabilities", International Journal of Engineering and Technology (IJET), Vol 5 No 1, pp:391- 397, 2013.

17. Joshi K., Ranjan R., Sravya E., Baig M.N.A. (2019) Design of Voice-Controlled Smart Wheelchair for Physically Challenged Persons. In: Abraham A., Dutta P., Mandal J., Bhattacharya A., Dutta S. (eds) Emerging Technologies in Data Mining and Information Security. Advances in Intelligent Systems and Computing, vol 814. Springer, Singapore

18. P. Dey, M. M. Hasan, S. Mostofa and A. I. Rana, "Smart wheelchair integrating head gesture navigation," 2019 International Conference on Robotics,Electrical and Signal Processing Techniques (ICREST), Dhaka, Bangladesh, 2019, pp. 329-334.

19. Amiel Hartman and Vidya K. Nandikolla, "Human-Machine Interface for a Smart Wheelchair," Journal of Robotics, vol. 2019, Article ID 4837058, 11 pages, 2019.

\section{AUTHORS PROFILE}

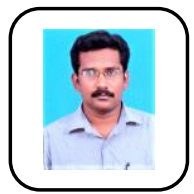

Srinivasan Selvaraj, has been working as an Associate Professor in the Department of Computer Science and Engineering since March 2012. He obtained his B.E(CSE) from Mohammad Sathak Engineering College and M.E(CSE) from Mepco Schlenk Engineering College. He obtained his Ph.D in Information and Communication Engineering at Anna University, Chennai in 2011. He has been in the teaching profession for the past 14 years. He has been handling courses to both UG and PG students. His areas of interest include Distributed Systems, Cloud Computing, Operating Systems and Data Mining. He has published 11 papers in International Journals and 12 papers in National and International conferences.. He is a life time members of Computer Society of India.

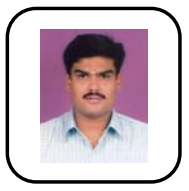

A.Gnanasekar has been working as a Associate Professor in Department of Computer Science and Engineering, since Septemper 2007. He has completed A.M.I.E. in the year 2002 at Institute of Engineering, Calcutta. He has completed M.Tech in the year 2005 at Bharath institution of Higher Education and Research (BIHER) Engineering College, Chennai. He received him Ph. D in Anna University, Chennai. He has 15 years of experience in teaching field and 6 years of experience in industry.His area of interest is Programming concept, Object Oriented Programming Languages, Internet Programming, Data Structure,Computer architectur,Web Technology.He has published 4 papers in various International Journals and Conferences.. He is a life time member of MIE.

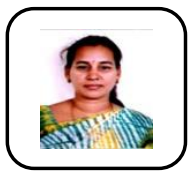

Dr. Pacha Shobha Rani is working as Associate Professor in Computer Science and Engineering of R.M.D.Engineering College,Chennai,Tamil Nadu, India. she pursued Degree in Bachelors of Engineering (CSE) from Bangalore University and Masters in Computer Science and Engineering from Anna University, Chennai. Presently she is pursuing her Ph.D., in the area of Ontology oriented Big Data. She has 5 years of working experience in the industrial field. She is in the teaching profession for the past 15 years. She also completed her ORACLE Certification. Her areas of interest include Data Warehousing and Data Mining, Big Data. She has published two research papers in journals and conferences. She has guided four Master of Engineering projects

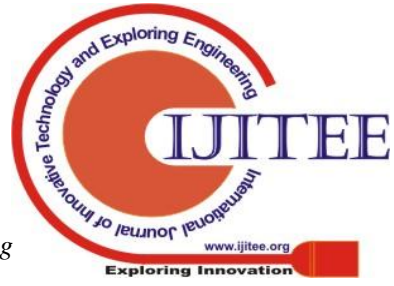


Dr.P.Ezhumalai, is the Professor and Head of the Department of Computer Science and Engineering at R.M.D Engineering College, Chennai. He has 25 Years of working experience in the teaching profession and 10 years of research experience.. He has written a book on Compute Programming. He has got National Citizenship Gold Medal Award for excellence from Global Economic Progress and Research Association, New Delhi. His research areas of expertise are Multi-core Architecture, Computer Networks, Theory of Computation and Compiler Design. Patent has been granted for his research "VIRTUAL JEWELLERY". He has published 32 papers in International/National journals and presented 16 papers in refereed International/National conferences. He is guiding M.E research scholars in his area. He has received Best Accredited Student Award on behalf of CSE students from CSI Chennai Chapter for three consecutive years 2012 to 2014 and 2016 $\mathrm{He}$ is a fellow member in CSI and IEI. 